\title{
A Large Keratocystic Odontogenic Tumor
}

\section{Saurabh Jolly, Jeevan Lata}

\begin{abstract}
Keratocystic odontogenic tumor (KCOT) is described as a benign uni- or multicystic, intraosseous tumor arising from the dental lamina or its remnants. Various treatment modalities available are decompression, marsupialization, enucleation, curettage, resection with and without continuity defect, and disarticulation. Among all of these treatment modalities for large keratocystic odontogenic tumor, marsupialization followed by enucleation is preferred owing to its conservative nature as it decreases the size of the cystic cavity gradually thus, it can save the adjacent anatomic structures. Here, we describe a case of large KCOT which was treated using conservative approach.
\end{abstract}

Keywords: Enucleation, Marsupialization, Keratocyst.

How to cite this article: Jolly S, Lata J. A Large Keratocystic Odontogenic Tumor. World J Dent 2013;4(2):138-143.

Source of support: Nil

Conflict of interest: None declared

\section{INTRODUCTION}

Odontogenic keratocyst (OKC) is now designated by the World Health Organization (WHO) as a keratocystic odontogenic tumor (KCOT) and is defined as 'a benign unior multicystic, intraosseous tumor of odontogenic origin, with a characteristic lining of parakeratinized stratified squamous epithelium and potential for aggressive, infiltrative behavior'. WHO recommends the term 'keratocystic odontogenic tumor' as it better reflects its neoplastic nature. ${ }^{1}$

This tumor, previously considered an odontogenic cyst was first reported by Philipsen in 1956 and attracted interest because of its specific histopathological features and its high recurrence rate. ${ }^{2,3}$

The OKC is thought to recur by the following mechanisms: ${ }^{4}$

1. Incomplete removal/residual cystic lesion gives rise to new cyst formation [microcysts (daughter cysts) or epithelial islands in the wall of the original cyst remain in the surrounding bone or soft tissue].

2. New keratocysts develop from epithelial offshoots of the basal layer of the oral epithelium.

3. Continuous formation of new cysts in patients with basal cell nevus syndrome.

Evidence derived from the examination of keratocysts from patients with the nevoid basal cell carcinoma (GorlinGoltz) syndrome (NBCCS) has suggested that the cysts may arise directly from dental lamina. ${ }^{5}$
KCOTs comprise approximately $11 \%$ of all cysts of the jaws.

The mandible is involved much more frequently than the maxilla. About half of the cases occur at the angle of the mandible and extend for varying distances into the ramus and forward into the body. There is no special relationship to a tooth. ${ }^{6}$

Patients with KCOTs may complain of pain, swelling or discharge. However, some are unaware of the lesion until they develop pathologic fractures; this occurs because KCOT tend to extend into the medullary cavity and clinically observable expansion of the bone occurs late. ${ }^{7}$

In case of maxilla, the aggressive behavior of the tumor penetrating the surrounding soft tissues, base of skull, orbit and infratemporal fossa have been reported. Sometimes KCOT occurs in association with basal cell nevus syndrome, and frequently there are multiple KCOTs.

KCOT radiographically, appears as a unilocular or multilocular lesion with a scalloped contour. These characteristics are suggestive but not considered an unequivocal proof for the definitive diagnosis of OKC because other lesions may exhibit similar features. ${ }^{8}$

Unilocular KCOT can be located periapically, simulating periapical cysts and can also present as a small radiolucency between teeth simulating a lateral periodontal cyst. Displacement of unerupted teeth and inferior and superior displacement of the mandibular canal can be observed.

The typical histologic features of the OKC include an epithelial lining of regular parakeratinized stratified squamous epithelium. The epithelium is thin, ranging from 6 to 10 cells thick, and lacks rete pegs, which produce the characteristic flat interface between the epithelium and connective tissue. Separation of the epithelium from the supporting connective tissue of the cyst is common and is caused by metalloproteinase-mediated degradation of collagen in the juxta-epithelial regions. ${ }^{9}$

The presence of small 'daughter' or 'satellite' cysts may be observed in the connective tissue wall of the KCOT. The tumors linings are thin and fragile, being difficult to enucleate in one piece.

In the literature there is a controversy regarding the treatment of KCOT: some surgeons advocate conservative therapies whereas others are in favor of an aggressive treatment. The goal is to choose the treatment modality that carries the lowest possible risk of recurrence and the least morbidity, while still eradicating the lesion. ${ }^{10}$ 
The treatment of KOT remains controversial and various surgical modalities have evolved in an attempt to decrease the recurrence rate, including enucleation, curettage, marsupialization, ${ }^{11,12}$ decompression, enucleation and treatment of the bony defect with Carnoy's solution, ${ }^{13}$ liquid nitrogen cryotherapy and resection with or without loss of jaw continuity. Patient characteristics are important determinants, including the patient's general health status, age, reliability for follow-up, and whether the patient has basal cell nevus syndrome.

\section{CASE REPORT}

A 36-year-old male patient reported to the Department of Oral and Maxillofacial Surgery, Punjab Government Dental College and Hospital, Amritsar, for evaluation of constant dull aching pain in left and right side of lower jaw radiating toward anterior region of mandible since 3 months. His medical history and general physical examination were unremarkable. Patient denied any use of tobacco. Intraoral examination showed a bicortical expansion present in mandible which was more evident in relation to the buccal cortex than the lingual cortex. Overlying mucosa was intact without any sign of sinus or fistula. No fluctuation or discharge was detected. The patient had fair oral hygiene with mild calculus deposits. Patient had full complement of healthy teeth except an occlusally carious left mandibular third molar. On electrical stimulation 37, 38 and 46 gave a negative response. Also these teeth were found to have grade 1 mobility. Tenderness on percussion was significantly noted in mandibular right first molar. The patient denied any neurosensory deficit associated with the inferior alveolar nerve. There was no clinical evidence of lymphadenopathy.

An orthopantomogram (OPG) was advised which revealed (Fig. 1), a large radiolucent lesion extending periapically from mesial of right mandibular third molar to distal of left mandibular third molar. There was evidence of bony septa on left side of the lesion in relation to left second premolar and first molar. The margins of the lytic lesions were found to have well defined scalloping. Additionally, supernumerary tooth was present at the lower border of mandible in relation to left mandibular canine and first premolar. All the teeth in the lesion showed blunting of root apices excluding the right mandibular third molar and mandibular incisor teeth. Under aseptic condition, aspiration of the lesion was done using a $5 \mathrm{ml}$ syringe and 18 gauze needle which yielded a thick cheesy odorless white material.

Based on clinical examination, radiographic interpretation and aspiration result, the lesion was provisionally

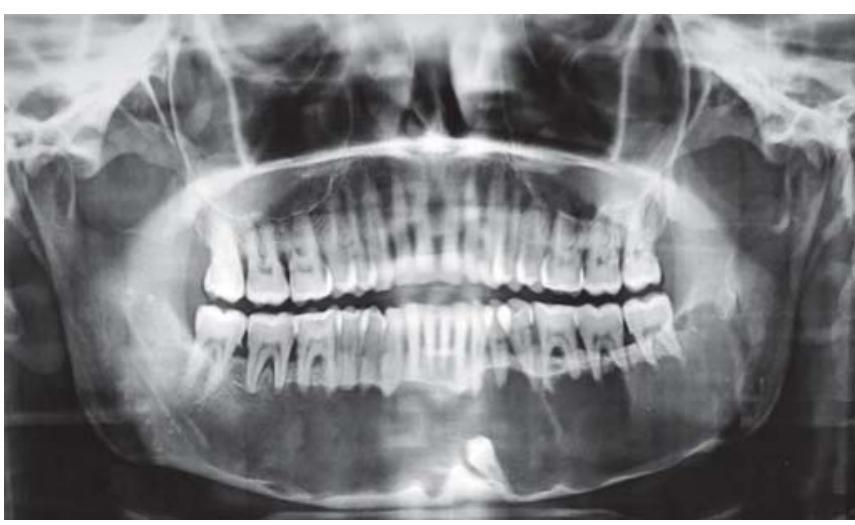

Fig. 1: Panoramic radiograph showing a large radiolucent lesion with an impacted supernumerary tooth in relation to inferior border of mandible

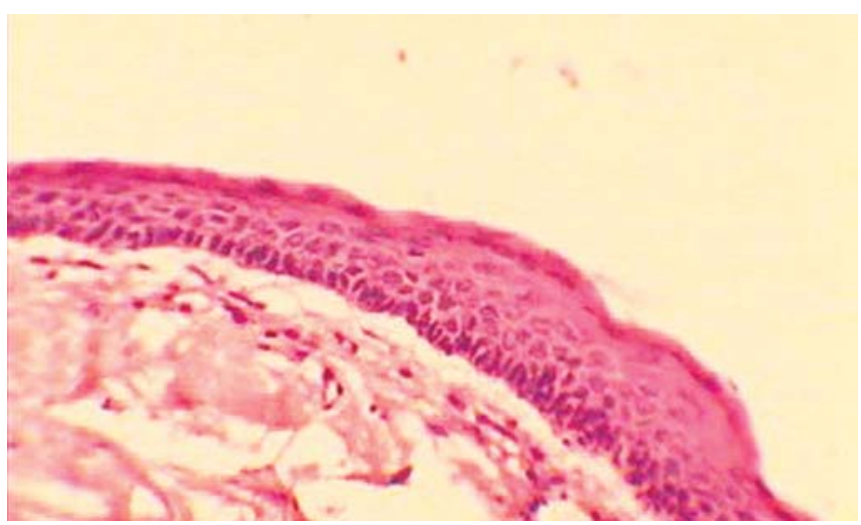

Fig. 2: Histopathologic view: Thin and regular parakeratinized stratified squamous epithelium without rete pegs and exhibiting the flat interface between the epithelium and connective tissue

diagnosed as OKC. Dentigerous cyst and cystic ameloblastoma were kept in differential diagnosis.

Marsupialization along with incisional biopsy was planned so as to decompress the cavity and for histopathological examination.

Routine laboratory investigations were within the normal limits. After taking an informed consent, patient was prepared for surgery under local anesthesia. Amoxicillin 1 gm was given (PO) 1 hour preoperatively to the patient. Using buccal sulcus approach, bilateral marsupialization was done in relation to right first and second mandibular premolar and left mandibular canine and first mandibular premolar. The window was created and a portion of exposed cystic lining was incised. The retrieved tissue was sent for histopathologically examination. Cavity was packed open with iodoform gauze which was changed at weekly intervals. Histopathologically (Fig. 2), it was confirmed to be a case of KCOT. After a period of 3 months, a repeat OPG (Fig. 3) was done which showed, marked reduction in the size of lesion. Hence, enucleation of the pathology was planned.

Again after taking an informed consent, patient was prepared for surgery under local anesthesia. Surgery was 


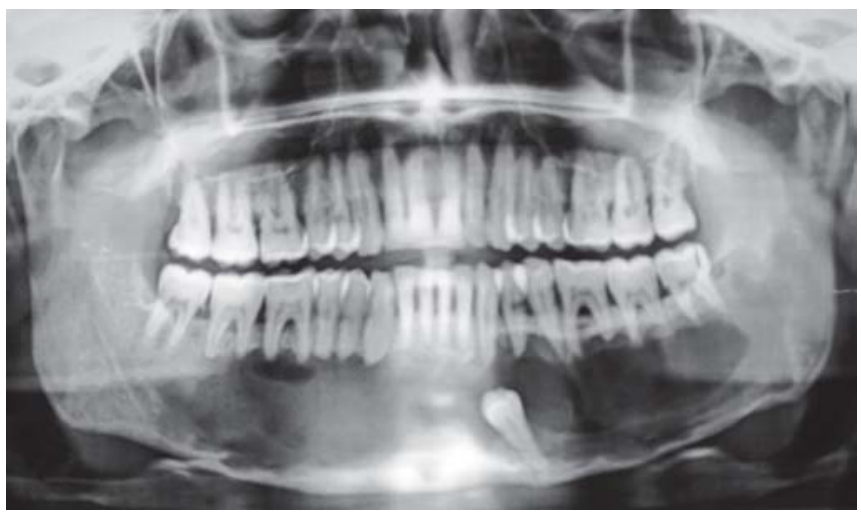

Fig. 3: Panoramic radiograph showing reduction in size of lesion while comparing with Figure 1

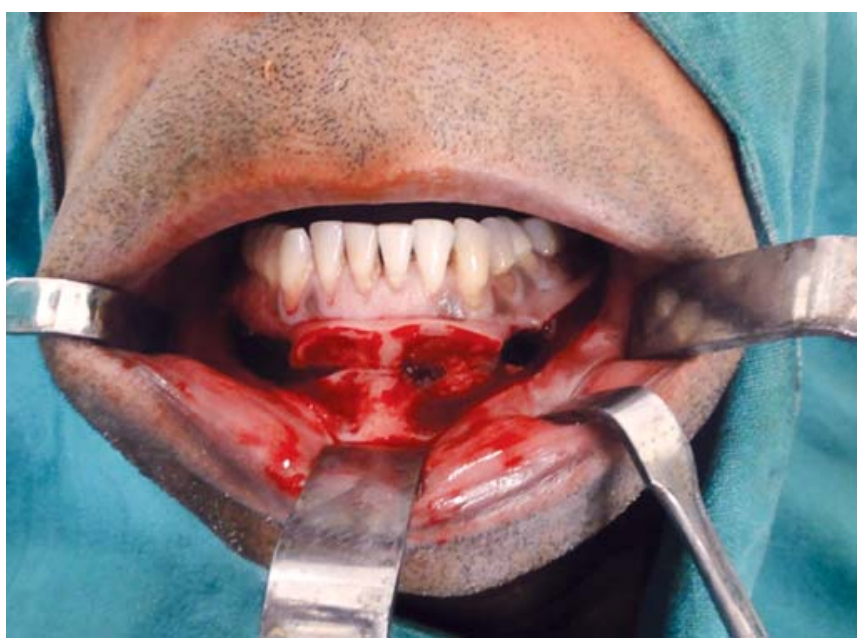

Fig. 5: An intraoperative view showing surgical exposure of lesion via degloving incision

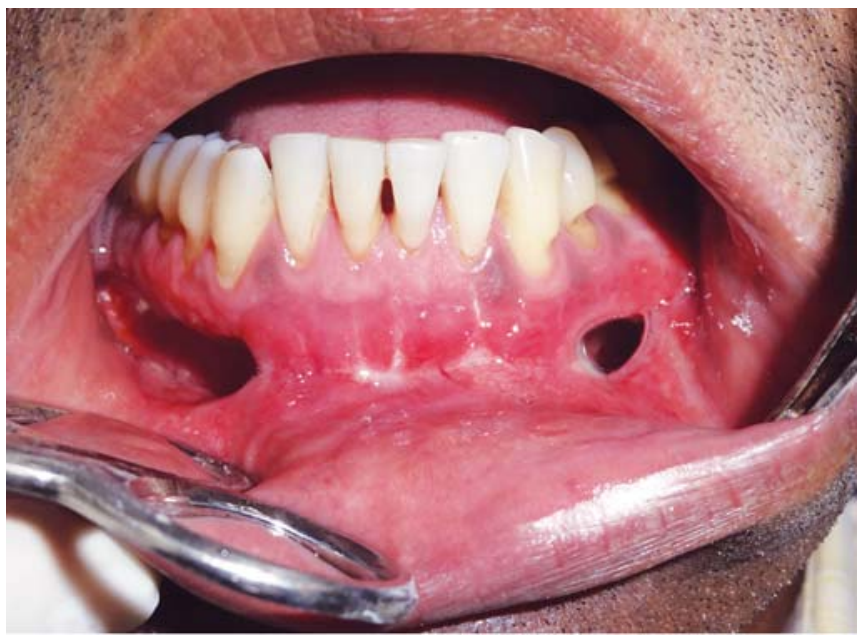

Fig. 7: Postoperative intraoral view after a period of 3 months

performed (Fig. 4) utilizing mandibular vestibular incision (Fig. 5). Intraoperatively, the mucosal lining was found to be relatively thickened which had occurred secondary to the effect of iodoform. Although KCOT has been reported to have a thin lining, but the periodic usage of iodoform

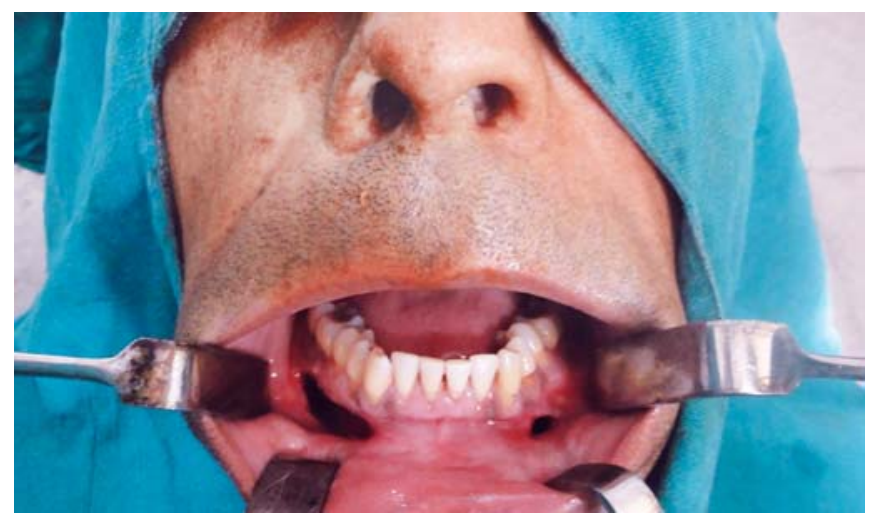

Fig. 4: Intraoral view showing the opening of marsupialized cavity

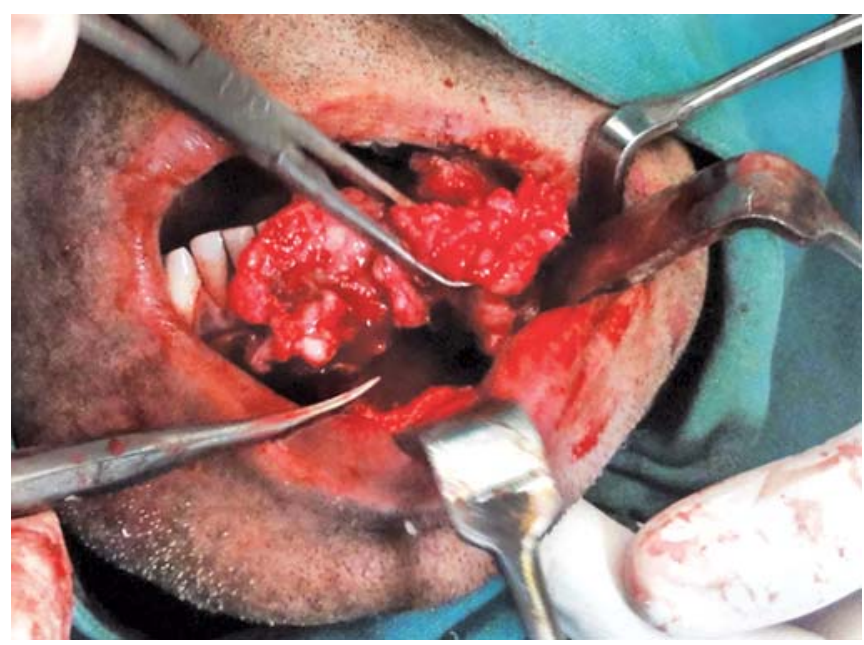

Fig. 6: Intraoperative view showing retrieval of cystic lining

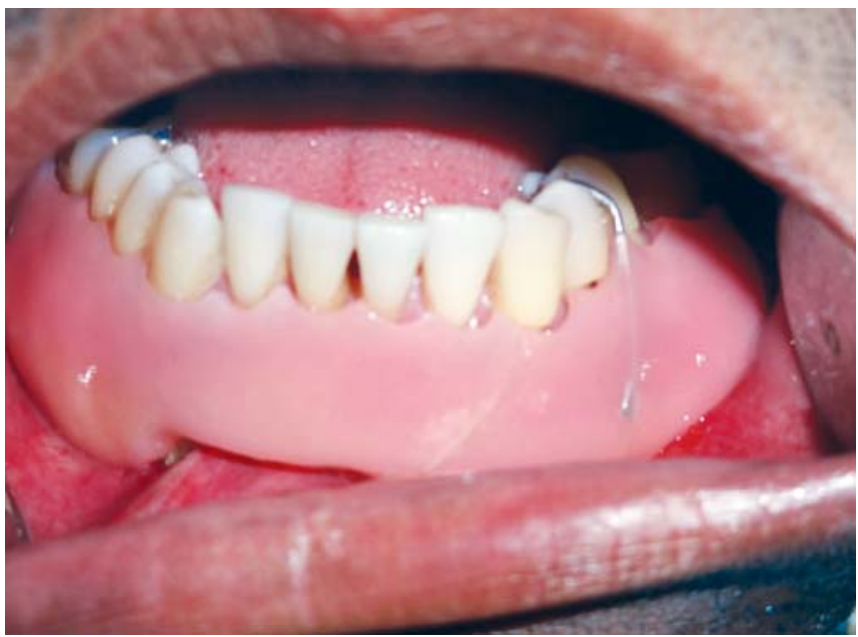

Fig. 8: Postoperative view along with removable prosthesis causing obliteration of cavity

transformed the lining into the thicker one. Thickened mucosal lining was easily separated from the bony walls and curetted out in toto. Figure 6 shows completely retrieved mucosal lining. The newly created cavity was again packed with iodoform roll gauze which was replaced at weekly 


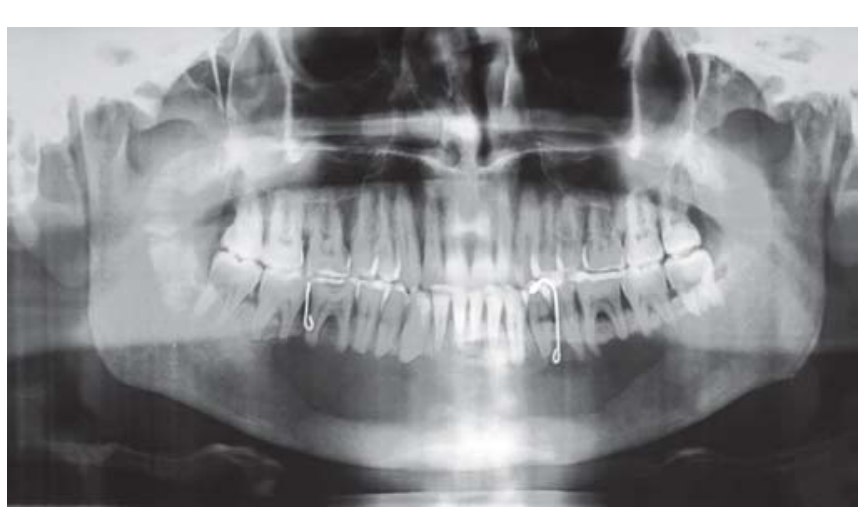

Fig. 9: Sufficient reduction in the size of lesion with new bone formation periapical to $38,47,48$ and adjacent ramus region along with anterior mandibular region. Compare this OPG with an image shown in Figure 1

intervals. Within a period of 6 weeks, the size of cavity (Fig. 7) was decreased and became self-cleansing. An obturator (Fig. 8) was designed which covered the cavity margins so the iodoform packing was no more required. Patient was kept on follow-up visits. Follow-up X-ray (Fig. 9) after 3 months interval showed significant reduction in the size of cavity. Clinically patient made an uneventful recovery except for mild paresthesia on right side of lower lip. Patient is advised long-term follow-up for any recurrence.

\section{Demerits of Surgery}

1. Patient had paresthesia of right and left inferior alveolar nerve for which patient was given methylcobalamin $1,500 \mu g$ daily. Presently left inferior alveolar nerve paresthesia is completely recovered. But paresthesia was persistent in relation to the right inferior alveolar nerve.

2. Frequent regular follow-up was inconvenient for the patient but patient shows good compliance in this aspect.

\section{DISCUSSION}

In 1956, Philipsen first described an odontogenic cyst with a keratinous epithelial lining and in 1962, Pindborg and Hansen described the typical histological features of OKC as follows.

The lining epithelium is usually very thin and uniform in thickness, with little or no evidence of rete ridges. There is also a well-defined basal cell layer and a thin spinous layer that often directly adjoins the palisaded arrangement. The cells of the spinous layer frequently exhibit intracellular edema. Keratinization is predominantly parakeratotic, but can also be orthokeratotic. The keratin layer is often corrugated. ${ }^{14}$

KCOTs have been reported to occur most commonly in the second and third decade of life but may be diagnosed in people of any age.
The tumor involves the mandible far more frequently than the maxilla. Shear in a study of 125 KCOTs, found 94 occurring in the mandible; another study with 183 KCOTs, revealed that $70.5 \%$ were also in the mandible. The tumor can occur in any part of the mandible, but the majority arises in the angle and ascending ramus. ${ }^{15}$

It is important to note that OKCs can be confused with inflammatory lesions since the patients with this type of cyst usually have inflammatory symptoms such as pain, swelling and drainage. ${ }^{16}$

Even being considered a benign tumor, some of the characteristics of KCOT make this pathology extremely serious. KCOT tend to recur, can reach considerable dimensions, and can arise close to delicate and important anatomical structures such as inferior alveolar nerve. All these characteristics had suggested, in the past, that an aggressive surgical approach should be followed in order to completely eradicate the lesion.

Radiographic appearance of OKCs is a unilocular or a multilocular radiolucency with scalloped and well-defined margins, mainly located in the aforementioned area of the mandible. In our case OKC present as large well-defined radiolucency having well-defined scalloped margins divided by thin septa. OKC can be associated with the crown of an included tooth; the cyst must be distinguished from a dentigerous cyst. Other cystic and neoplastic diseases, such as traumatic bone cyst, lateral periodontal cyst, central giant cell granuloma, fissural cysts, minimally calcifying odontogenic cyst, radicular cyst, arteriovenous malformation, benign bone tumors, ameloblastoma, adenomatoid odontogenic tumor, ameloblastic fibroma and plasmacytoma can present with the same radiologic features.

The treatment of KCOT remains controversial and various surgical modalities have evolved in an attempt to decrease the recurrence rate, including enucleation, curettage, marsupialization, decompression, enucleation and treatment of the bony defect with Carnoy's solution, liquid nitrogen cryotherapy and resection with or without loss of jaw continuity. Patient characteristics are important determinants, including the patient's general health status, age, reliability for follow-up, and whether the patient has basal cell nevus syndrome.

Treatment of the cyst with Carnoy's solution before enucleation is helpful to prevent any remnants that are left from developing into recurrence, but it may also damage adjacent bone and nerve fibers. Application of Carnoy's solution before enucleation for 10 to 15 minutes is useful. If the inferior alveolar nerve is visible in the bony cavity after enucleation, Carnoy's solution should not be directly applied to this area or applied beyond 3 minutes. ${ }^{17}$ 
Carnoy's solution is a mixture of absolute alcohol $6 \mathrm{ml}$, chloroform $3 \mathrm{ml}$, glacial acetic acid $1 \mathrm{ml}$, and ferric chloride $1 \mathrm{gm}$, that penetrates bone to a predictable, time-dependent depth without injuring the neurovascular structures. A 5-minute application penetrates bone to a depth of $1.54 \mathrm{~mm}$, nerve to a depth of $0.15 \mathrm{~mm}$, and mucosa to a depth of $0.51 \mathrm{~mm}^{18}$

The main advantage of the conservative treatment is the preservation of bone structure, woven soft and teeth associated OKC, fact that it is covered of great importance if we consider that most of the patients are young. Time of duration of the decompression treatment (1-14 months) is one of the disadvantages of this technique. In fact, this is one of the main causes of abandonment of the treatment by the patient because of loss of interest in proper irrigation treatment and attendance of periodic controls. These procedures are less traumatic for the patient, eliminating medication and hospitalization expenses, and in most cases, avoid the need to accomplish reconstruction through grafts or extensive reconstructions that require general anesthesia and hospitalization.

Tooth vitality was revised in relation to 37,38 and 46 following the surgery, which reveals vital response of teeth on electrical stimulation. This fact was supported that there may be temporary absence of vital response in adjacent teeth associated with large cysts because of pressure interference with sensory transmission from the pulp..$^{19}$

In contrast, simple decompression of a keratocyst is a relatively straightforward procedure that involves the placement of a drain into the cyst to allow it to decrease in size before definitive enucleation, which removes all of the cystic epithelium by whatever means chosen. This procedure is followed by immediate or secondary bone grafting as appropriate. The technique of decompression theoretically should be allowed to proceed until important structures can be preserved predictably preserved (adjacent teeth, nerves, sinus and nasal cavity) and bone grafting is not required. There is a general feeling that cysts larger than 4 to $5 \mathrm{~cm}$ in diameter in adults probably should be bone grafted; in children, cysts larger than 6 or $7 \mathrm{~cm}$ may require grafting to ensure adequate bone bulk for cosmesis and possible implant insertion.

Analysis of recurrence rate of each method seems to suggest that it is highest with marsupialization (percent) compared with 16.7 to $18.2 \%$ in cases of enucleation. The recurrence rate does not appear to be significantly different between enucleation followed by primary closure (18.2\%) and secondary granulation $(16.7 \%){ }^{20}$

Resection is the only predictably curative procedure for patients with OKCs that offers a recurrence rate approaching zero. The concern with this method is the functional and esthetic morbidity associated with reconstructing continuity defects of the mandible and maxilla. Advances in mandibular reconstruction, microvascular surgery, and dental implants during the last 2 decades, however, have made this aggressive treatment modality less foreboding. The need for resection might be answered by this question: is the reconstructive use of the residual osseous structure less than what could be provided entirely from distant tissue? If so, the resection and composite reconstruction would be appropriate. $^{11}$

In the present case the initial treatment planning was marsupialization followed by definitive removal by enucleation and curettage after the tumor volume reduction was reached. Its well established in the literature that enucleation has the highest recurrence rates in the treatment of KCOTs and this is partially explained by the characteristic thin and friable lining of the KCOT that makes removal of the tumor as a single piece difficult undertaking.

\section{CONCLUSION}

In light of the previous clinical trials, this case report outlines that large multilocular keratocysts might be treated with a conservative approach, the only disadvantages being the extended therapeutic time and the frequent recurrence of lesion.

\section{REFERENCES}

1. Barnes L, Eveson JW, Reichart P, Sidransky D. Pathology and genetics of head and neck tumours. WHO classification of tumour series. Lyon: IARC Press 2005:1-430.

2. González-Alva P, Tanaka A, Oku Y, Yoshizawa D, Itoh S, Sakashita H, et al. Keratocystic odontogenic tumor: A retrospective study of 183 cases. J Oral Sci 2008;50(2):205-12.

3. Maurette PE, Jorge J, Moraes M. Conservative treatment protocol of odontogenic keratocyst: A preliminary study. J Oral Maxillofac Surg 2006; 64(3):379-83.

4. Giuliani M, Grossi GB, Lajolo C, Bisceglia M, Herb KE. Conservative management of a large odontogenic keratocyst: Report of a case and review of the literature. J Oral Maxillofac Surg 2006;64(2):308-16.

5. Shear M. Odontogenic keratocysts: Natural history and immunohistochemistry. Oral Maxillofacial Surg Clin N Am 2003;15:347-62.

6. Jordan RC. Histology and ultrastructural features of the odontogenic keratocyst. Oral Maxillofacial Surg Clin N Am 2003 Aug;15(3):325-33.

7. Shear M. Odontogenic keratocyst: Clinical features. Oral Maxillofac Surg Clin N Am 2003;15(3):335-45.

8. Stoelinga PJ. Long-term follow-up on keratocysts treated according to a defined protocol. Int J Oral Maxillofac Surg 2001 Feb;30(1):14-25.

9. Sembronio S, Albiero AM, Zerman N, Costa F, Politi M. Endoscopically assisted enucleation and curettage of large 
mandibular odontogenic keratocyst. Oral Surg Oral Med Oral Pathol Oral Radiol Endod 2009;107(2):193-96.

10. Kolokythas A, Fernandes R, Pazoki A, Ord RA. Odontogenic keratocyst: To decompress or not to decompress? A comparative study of decompression and enucleation versus resection/ peripheral ostectomy. J Oral Maxillofac Surg 2007;65(4): 640-44.

11. Zhao YF, Wei JZ, Wang WP. Treatment of odontogenic keratocysts: A follow-up of 255 Chinese patients. Oral Surg Oral Med Oral Pathol Oral Radiol Endod 2002;94:151-56.

12. Pogrel MA. Decompression and marsupialization as a treatment for the odontogenic keratocyst. Oral Maxillofac Surg Clin N Am 2003;15(3):415-27.

13. Stoelinga PJW. The treatment of odontogenic keratocysts by excision of the overlying attached mucosa, enucleation, and treatment of the bony defect with Carnoy solution. J Oral Maxillofac Surg 2005;63(11):1662-66.

14. Kwon HI, Lim WB, Kim JS, Ko YJ, Kim IA, Yoon SJ, et al. Odontogenic keratocyst associated with an ectopic tooth in the maxillary sinus: A report of two cases and a review of the literature. Korean J Pathol 2011;45(S1):S5-10.

15. Contar CMM, Pompermayer A, Souza PHC, Propst GB, Costa MBD, Chagas PR. Keratocystic odontogenic tumor: Case report. Odonto 2011;19(37):25-32.

16. Tsukamoto G, Sasaki A, Akiyama T, Ishikawa T, Kishimoto K, Nishiyama A, et al. A radiologic analysis of dentigerous cysts and odontogenic keratocysts associated with a mandibular third molar. Oral Surg Oral Med Oral Pathol Oral Radiol Endod 2001;91(6):743-47.

17. Frerich B, Cornelius CP, Weitholter H. Critical time of exposure of the rabbit inferior alveolar nerve to Carnoy's solution. J Oral Maxillofac Surg 1994;52:599-606.

18. Walia A, Binjoo N. Decompression with marsupialization and enucleation of the keratocyst: A clinical study. JIDA 2012;6(1):25-29.

19. Laskin DM. Oral and maxillofacial surgery. St Louis, MO: CV Mosby 1985;2:431.

20. Ong ST, Siar CH. Odontogenic keratocysts in a Malaysian population: Clinical, radiological and histological considerations. Annals Dent Univ Malaya 1995;2:9-14.

\section{ABOUT THE AUTHORS}

\section{Saurabh Jolly (Corresponding Author)}

Postgraduate Student, Department of Oral and Maxillofacial Surgery Punjab Government Dental College and Hospital, Amritsar, Punjab, India e-mail: drsaurabh.jolly@yahoo.com

\section{Jeevan Lata}

Professor and Head, Department of Oral and Maxillofacial Surgery Punjab Government Dental College and Hospital, Amritsar, Punjab India 